FOMENTO DE LA ACTITUD EMPRENDEDORA, EN EL AMBIENTE UNIVERSITARIO PARA LA CREACIÓN DE MIPYMES. EL CASO DE POSGRADO DE LA DACEA-UJAT.

\title{
FOMENTO DE LA ACTITUD EMPRENDEDORA, EN EL AMBIENTE UNIVERSITARIO PARA LA CREACIÓN DE MIPYMES. EL CASO DE POSGRADO DE LA DACEA-UJAT.
}

\author{
María Adisela Hernández Morales*
}

\section{RESUMEN}

Objetivo: Analizar la importancia del fomento de la actitud emprendedora en alumnos de Posgrado de la DACEA-UJAT, y que influya para la creación de MIPYMES basado en un enfoque de modelos de negocio.

Material y método: El estudio se dirigió a alumnos de Licenciatura y sobre todo de Posgrado Universitarios de la DACEA-UJAT con visión para la creación de empresas mismas que pueden ser del sector privado pero con responsabilidad social $u$ organizaciones sociales que solucionen alguna problemática del entorno de algún grupo vulnerable; la propuesta se fundamentó en resultados de una muestra de 73 estudiantes de la población total de alumnos inscritos en las diferentes maestrías ofertadas en la División Académica de Ciencias Económico Administrativas (DACEA), que se obtuvo en base a un muestreo aleatorio simple. Se elaboraron instrumentos de recolección de datos, un cuestionario de preguntas categorizadas y cerradas y una guía de entrevista a funcionarios del Programa Emprendedores de UJAT. Se abordaron algunos enfoques teóricos siguiendo los canales de Joseph Schumpeter, las guías del Lienzo del Modelo de Negocios, los antecedentes y experiencias de personajes impulsores de la cultura emprendedora de esta Casa de Estudios.

Resultados: Los elementos de fomento a la actitud emprendedora en la Institución son los profesores miembros de las Comisiones Divisionales y los alumnos de licenciaturas, los programas de maestría no entran en esta dinámica al cien por ciento. Sin embargo, los resultados preliminares de una prueba piloto, muestran que el $78 \%$ de los respondientes tiene ideas para echar a andar un negocio y un 92\% mencionó que le gustaría contar con apoyos que le ayuden a generar una microempresa.

Conclusiones: Se necesita trabajar con modelos de negocios, que formen y fomenten el espíritu emprendedor, forjado en el ambiente académico de esta Universidad a través del

`Maestra en Administración. División Académica de Ciencias Económico Administrativas. Universidad de Juárez Autónoma de Tabasco. 
FOMENTO DE LA ACTITUD EMPRENDEDORA, EN EL AMBIENTE UNIVERSITARIO PARA LA CREACIÓN DE MIPYMES. EL CASO DE POSGRADO DE LA DACEA-UJAT.

Programa Educativo del Posgrado. Dado que los estudiantes, muestran mucho interés en crear su propia fuente de empleo al concluir el programa de estudios.

Palabras clave: creación de MIPYMES, espíritu emprendedor, actitud emprendedora, modelo de negocio, emprendedurismo.

\section{ABSTRACT}

Objective: Analyze the importance of the promotion of the entrepreneurial attitude in students Graduate DACEA-UJAT, and to influence for the creation of small businesses an approach based on business models.

Material and method: The study Undergraduate students and especially the Graduate College UJAT-DACEA with a vision for the creation of companies, which can be private but social responsibility or social organizations that solve some problems of an environment vulnerable group; the proposal was based on results from a sample of 73 students of the total population of students enrolled in the different masters offered in the División Académica de Ciencias Económico Administrativas (DACEA), which was obtained based on simple random sampling. Data collection instruments, questionnaire categorized and closed questions and an interview guide officials UJAT Entrepreneurs Program were developed. Some theoretical approaches are discussed following the analysis of Joseph Schumpeter, guides Business Model Canvas, background and experiences of characters drives the entrepreneurial culture of this University.

Results: The elements of promotion of entrepreneurship attitude in the institution teachers are members of Divisional Committees and students of bachelor's, master's programs do not come into this dynamic one hundred percent. However, the preliminary results of a pilot test, show that $78 \%$ of respondents have ideas to start a business and $92 \%$ said they would like to have support to help you generate a microenterprise.

Conclusions: It needs to work with business models and to form and promote entrepreneurship, forged in the academic environment of the University through the Graduate Education Program. Since students, show much interest in creating their own source of employment at the end of the study program. 
FOMENTO DE LA ACTITUD EMPRENDEDORA, EN EL AMBIENTE UNIVERSITARIO PARA LA CREACIÓN DE MIPYMES. EL CASO DE POSGRADO DE LA DACEA-UJAT.

Keywords:

Creation of small businesses, entrepreneurship, entrepreneurial attitude, business model.

DIRECCIÓN PARA RECIBIR CORRESPONDENCIA: Correo electrónico: mariadisela@hotmail.com 
La figura del emprendedor se remonta desde las primeras agrupaciones humanas; somos una especie emprendedora, porque en el transcurso de la existencia se han generado patrones de conducta relacionados con la creación y la innovación, siempre en la búsqueda del desarrollo y progreso social. Emprender es simplemente iniciar algo con perseverancia y tenacidad. En este proceso, posiblemente largo y complejo se necesita también, disposición, voluntad, potencial y primordialmente ideas, para transformarlas en lo que vendría siendo el concepto de negocio.

Una persona al egresar de la universidad o al estudiar un posgrado muestra mucho interés en crear su propia fuente de empleo, se esmera y lo logra. Sin embargo, ocurren algunas circunstancias que lo orillan a cerrar su negocio, por ejemplo, no tomó en cuenta las condiciones del mercado, su idea de negocio no fue la adecuada, tuvo poca orientación, etcétera. Y por alguna de estas situaciones no sobrevivió a más de dos años en su emprendimiento. En el otro caso, cuando aún no se ha creado el negocio, cuando sólo son ideas, la mayoría de las veces, queda en esa fase, de las ideas. Aunado a esto, el estímulo al desarrollo de actitudes y habilidades emprendedoras en los alumnos de Posgrado ha sido escaso. Tampoco se ha trabajado con modelos de negocios sino con ideas. Tal vez porque no se ha analizado con profundidad el modo en que se crea un negocio.

En la actualidad, la formación universitaria implica generar propuestas orientadas al fomento del desarrollo económico local, por lo que "la utilización de las ideas apropiadas se convierte en la principal fuente de ventaja competitiva para los individuos y la organización en la época actual" (Cabrera, 2005). Por lo anterior, la creación de una MIPYME ${ }^{1}$, es el resultado de la transformación de las ideas, considerando las condiciones del entorno y combinando la actitud emprendedora en la formación del estudiante Universitario.

La Universidad Juárez Autónoma de Tabasco, desde el año 2001 incorpora la formación de emprendedores a sus planes de estudios, coadyuvando en esa perspectiva para con el profesionista egresado de sus programas. Hoy en día, es necesario que en esta

\footnotetext{
${ }^{1}$ MIPYME: Micro, pequeña y mediana empresa, legalmente constituida, con base en la estratificación establecida por la Secretaría de Economía, de común acuerdo con la Secretaría de Hacienda y Crédito Público y publicada en el Diario Oficial de la Federación del 18 de enero de 2012. (Diario Oficial de la Federación, 2013) Consultar la web: http://www.dof.gob.mx/
} 
formación se considere la responsabilidad social en los proyectos productivos, además de fortalecer la creación de empresas con el compromiso de responder a la solidaridad que la dinámica actual demanda.

El presente trabajo, muestra observaciones y resultados de un estudio del Área de Posgrado de la División de Ciencias Económico Administrativas, DACEA en la Universidad Juárez Autónoma de Tabasco, UJAT. Con la intención de conocer las habilidades emprendedoras de los alumnos de Maestrías del área de negocios; así como los apoyos que existen para los alumnos por parte de la Institución, como son la Incubadora de Empresas de Tecnología Intermedia, concursos y ferias de negocios, entre otros.

\section{Revisión Literaria}

El término emprendedurismo se utiliza para referirse a los factores sociológicos y psicológicos (Stoner, Freeman \& Gilbert,1996), relacionados fuertemente a una motivación para iniciar una actividad empresarial. Tener un espíritu emprendedor, significa simplemente que se está predispuesto hacia la actividad emprendedora y que, hay una probabilidad sustancialmente creciente que algún día se elegirá comenzar con una actividad emprendedora y se tendrá éxito, tal como lo sugiere Gutiérrez (2011). El individuo que figura en esta actividad, es el emprendedor. Según el Diccionario de la Real Academia de la Lengua Española, es aquella persona que "emprende con resolución acciones dificultosas o azarosas". En el ámbito empresarial puede definirse como acometer y comenzar un negocio, con empeño, especialmente si encierra dificultad o peligro.

Investigaciones y programas pioneros en el tema del emprendimiento vienen ratificando el énfasis economicista y social del fenómeno, su perspectiva enfocado a la creación de empresa, en atender dificultades de desempleo y de generación de ingresos, y finalmente su implementación en los programas educativos.

Hasta principios del siglo XX, no se reconocía en la economía el rol del emprendedor y del riesgo. Joseph Schumpeter (1883-1950), brillante economista austriaco-estadounidense, escribió a principios de los años cuarenta, que un sistema económico sano, con frecuencia, estaba estimulado por una "destrucción creativa", -significa que la economía 
funciona en base a ciclos perpetuos de destrucción de productos y servicios viejos y menos eficientes que son reemplazados con nuevos y más eficientes-. En su clásico libro de La Teoría del Desarrollo Económico, destaca el aporte del emprendimiento al desarrollo y provee una de las primeras definiciones de emprendedor, "aquellos cuya función es llevar adelante nuevas combinaciones a las que llamamos empresas; su función característica consistía en romper moldes y crear nuevas tradiciones" (Anthony \& Nitin, 2007).

El ilustre economista reconoció al emprendedor como centro del sistema económico. Introdujo el concepto de innovación como la base de una persona con actitudes emprendedoras; así mismo, manifiesta que los emprendedores son quienes innovan en los siguientes aspectos: 1. La introducción de buenos y mejores servicios y productos (o el mejoramiento de uno existente); 2. Nuevos métodos de producción; 3. La apertura de nuevos mercados; 4. La conquista de nuevas fuentes de suministros de materias primas; y 5. La creación de una nueva organización industrial, (Gutiérrez, 2011).

Para Schumpeter (Ramírez, 2011), las invenciones e innovaciones son la clave del crecimiento económico y quienes implementan ese cambio de manera práctica son los emprendedores. Y es que para él, un emprendedor era "una especie individualista de súper hombre", los famosos entrepreneurs o schumpeterianos que establecían nuevas formas de producción, que combinaban factores de una forma novedosa o realizaban nuevas combinaciones.

No cabe duda que los trabajos de Joseph Schumpeter sobre el emprendimiento y la innovación y su efecto en el desarrollo económico, constituyen una contribución teórica de mucha importancia. A pesar de lo difícil que resulte ser innovador y lograr incursionar en el mercado, lo que verdaderamente importa es poseer una idea nueva de negocio, trascender de la idea a concepto de negocio, y arriesgarse, cambiando la rutina en el mercado.

En este proceso de emprender, el foco inicial es la idea de negocio, para la cual será necesario el uso de diversas metodologías que posibilitan el surgimiento de muchas, variadas e inusuales ideas de negocios, productos, etc., y a su vez, se transformen en conceptos, entre las cuales, se recomiendan la de SCAMPER (sustituir, combinar, 
adaptar, modificar, ponerle otros usos, eliminar, reorganizar), rediseño (comparar atributos de un producto para potencializar los positivos y neutralizar los negativos o los que no valora el cliente), lentes de creatividad (cuestionar creencias, detectar tendencias, ver necesidades y aprovechar competencias), modelos de negocio (innovación en modelos de negocio por encima de innovación en procesos, productos o relación con el cliente), y retos y concursos (estrategias para capturar ideas).

Estas metodologías permiten desde la generación de ideas, hasta la transformación de estas a conceptos de negocios; y que por supuesto, es lo que se debe ofrecer para que el cliente perciba el valor agregado del producto o servicio (Mesa, 2011).

El modelo de negocio es la forma en que se organizan personas y los recursos para capturar esa oportunidad a través de la idea. Describe los fundamentos de cómo una organización crea, desarrolla y captura valor para obtener los objetivos empresariales. A diferencia de un plan de empresa, un modelo de negocio es un prototipo dinámico, basado en hipótesis que se testean y cambian en función de los test (Osterwalder \& Pigneur, 2011).

En el lienzo del modelo de negocio (esquema 1), creado por Alexander Osterwalder, se representa por medio de un diagrama denominado "lienzo", una antología de nueve módulos, con sus análisis y reglas de interrelaciones entre ellos, que constituye una instrumento que facilita y da claridad sobre las actividades a realizar para el diseño, evaluación e innovación de modelos de negocio; es decir, la manera en como el negocio compra y vende bienes o servicios para obtener un beneficio. La división de sus nueve módulos, refleja cuál es la lógica que sigue una empresa para conseguir ingresos, cubriendo los pilares básicos: clientes, oferta, infraestructura y finanzas, que van de la mano de las cuatro preguntas básicas del emprendedor: el qué, el cómo, el quién y el cuánto. 


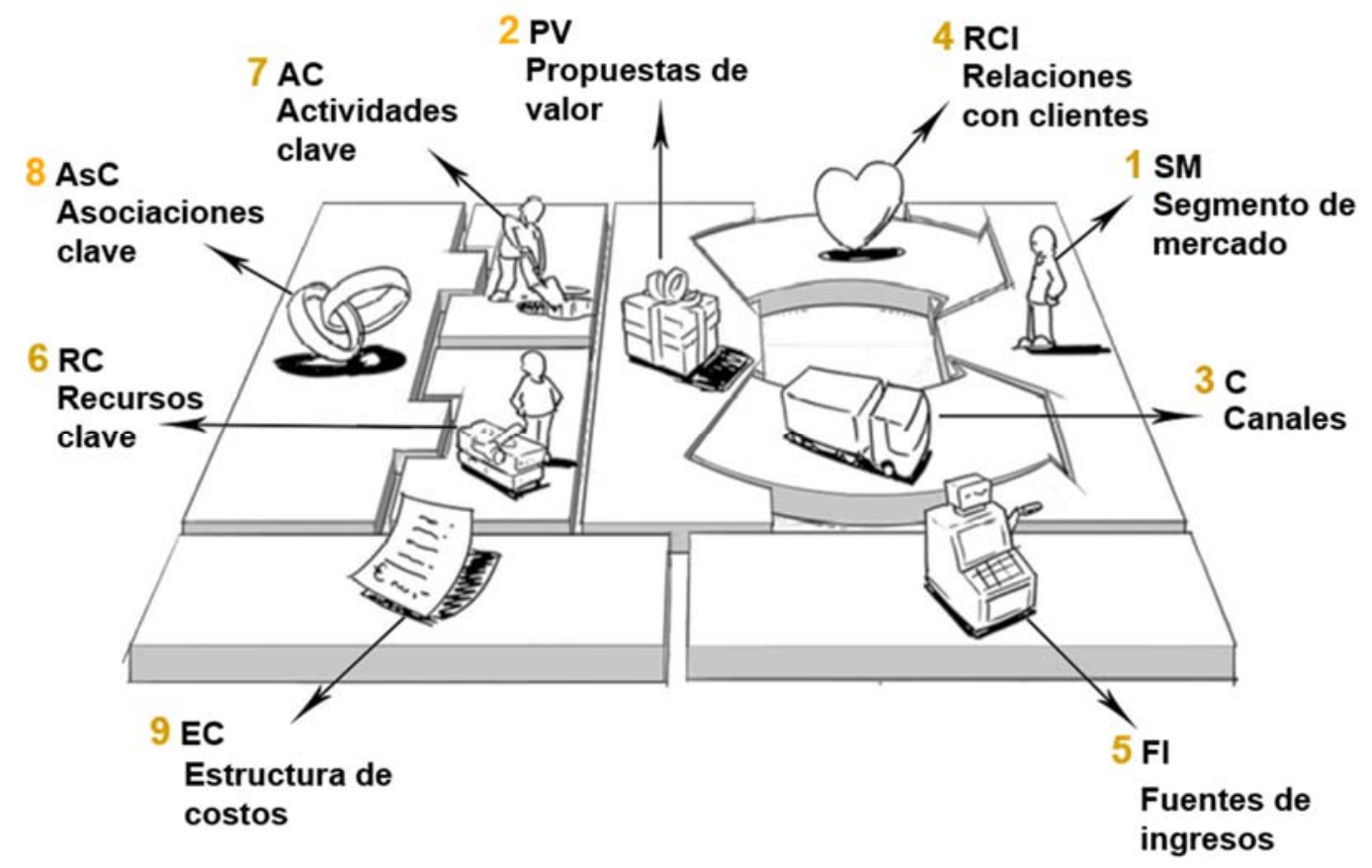

Esquema 1. La ontología de modelos de negocio propuesta por Osterwalder.

Fuente: Osterwalder y Pigneur (2011).

Los nueve módulos del Lienzo de Modelo de Negocio consisten en:

1. El segmento de mercado. Una empresa atiende a uno o varios segmentos de mercado, se tiene que identificar cuáles serán los posibles mercados donde se ofrecerá el producto o servicio.

2. Las propuestas de valor. Su objetivo es solucionar, los problemas de los clientes y satisfacer sus necesidades mediante propuestas de valor. Que se ofrece, que otros no lo hacen.

3. Los canales. Las propuestas de valor llegan a los clientes a través de los canales de comunicación, distribución y venta. Se describe la manera que se llega a comunicar con los segmentos del cliente y la forma en la que entrega una propuesta de valor a la misma.

4. Las relaciones con los clientes. Se estudia cómo se relacionarán los clientes, al establecer y mantener de forma independiente en los diferentes segmentos de mercado. Se debe aclarar el tipo de relación que quiere establecer con cada segmento de cliente. 
5. Las fuentes de ingresos. La ganancia es el resultado de la resta de los ingresos y gastos. Se generan cuando los clientes adquieren las propuestas de valor, es decir, son el resultado de propuestas de valor ofrecidas con éxito a los clientes.

6. Los recursos clave. Son los medios o activos necesarios para ofrecer, proporcionar y entregar los elementos antes descriptos. Estos recursos se relacionan directamente con el siguiente modulo, las actividades clave, pues son los primeros aquellos que se transforman, a través de las actividades, en las propuestas de valor de la empresa. En el modelo de negocio de Osterwalder, los recursos clave se clasifican en físicos, intelectuales, humanos y financieros.

7. Las actividades clave. Mediante la realización de una serie de actividades clave. Se determina cuales se tendrán que llevar a cabo.

8. Las asociaciones clave. Dado que algunas actividades se externalizan y determinados recursos se adquieren fuera y que hacen que el modelo de negocio funcione. Son necesarias para crear y ofrecer la propuesta de valor, llegar a mercados, establecer relaciones con clientes y obtener ganancias. Hay cuatro tipos de alianzas: alianzas entre no competidores, cooperación entre competidores, empresa conjunta para la creación de nuevos negocios y relaciones entre comprador y distribuidor. Por medio de la alianza se asegura la adquisición del recurso que se necesita.

9. La estructura de costos. Los diferentes elementos del modelo de negocio dan como resultado la estructura de costos como los costos fijos, variable y economías de escala y de alcance.

El lienzo de Osterwalder tiene algunos beneficios como, por ejemplo, es muy amigable por la facilidad, el gran poder visual y de síntesis que tiene. Permite un lenguaje común para describir, visualizar, analizar, evaluar, y cambiar los modelos de negocios, productos o servicios. Además, puede ser aplicado desde una MIPYME hasta la gran empresa.

Finalmente, el proceso del diseño del modelo de negocios es parte de la estrategia de negocios, por lo que es de vital importancia estructurar este tipo de recursos para conocer a profundidad cómo opera una empresa, sus fortalezas y debilidades. Sin embargo, 
encontrar una idea es el primer paso en el proceso de convertir la creatividad del emprendedor en un concepto de negocio (la esencia misma del negocio) y el modelo de negocio es la forma en que se organizan las personas y los recursos para capturar ese concepto a través de la idea.

\section{MATERIAL Y MÉTODO}

La investigación se centró en la unidad de Posgrado de la División Académica de Ciencias Económico Administrativas. La población total se constituyó de 91 alumnos inscritos en las diferentes maestrías que se ofertan en la División, tales como Administración, Administración Pública y Fiscalización de la Gestión Pública.

Para la obtención del tamaño de la muestra, se hizo uso de la siguiente fórmula para poblaciones finitas (menor o igual a 100,000):

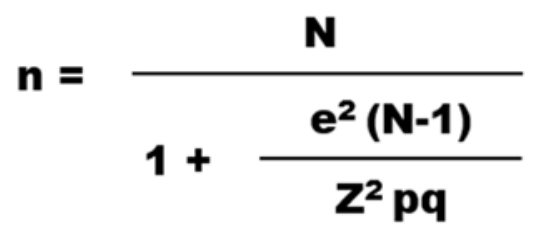

Dónde: $\mathrm{N}$ es el tamaño de la población; $\mathrm{n}$ es el tamaño de la muestra; $\mathrm{Z}$ es el nivel de confianza; $p$ es la variabilidad positiva; $q$ es la variabilidad negativa y e es la precisión o error. Se aplicó una prueba piloto consistente en 38 cuestionarios, de la cual se obtuvo: $p=0.6579$ y $q=0.3421$. Considerando un nivel de confianza de $95 \%$ y un error permisible de $5 \%$, de una población de 91 estudiantes se tiene:

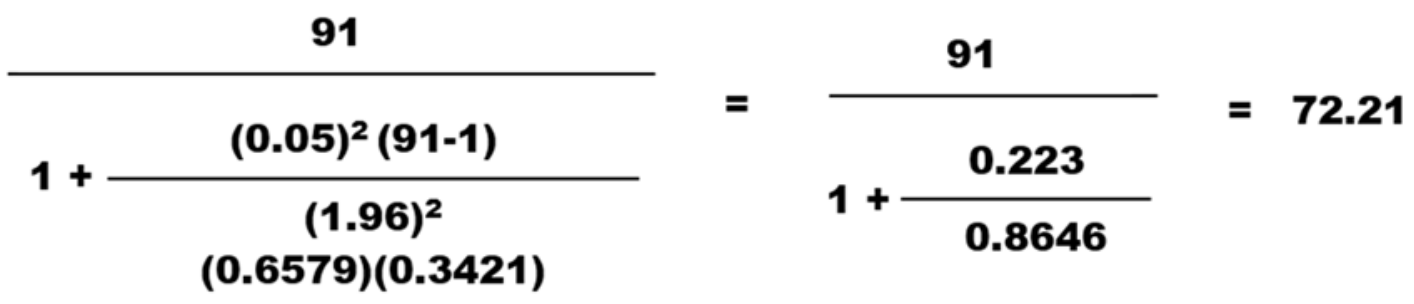

Por lo tanto $n=73$ alumnos de posgrado. $Y$ a esta muestra se aplicó un cuestionario de preguntas categorizadas y cerradas y una guía de entrevista a funcionarios responsables de la ejecución del Programa Emprendedores de UJAT, por lo que se considera un método mixto por la utilización de técnicas de análisis cuantitativas y cualitativas. 
Se analizaron los datos, realizando los procesos estadísticos correspondientes y se consideraron también los hallazgos de entrevistas a funcionarios del Programa de Emprendedores de la UJAT. Además de la revisión de fuentes de libros y publicaciones impresas y online acerca de la teoría de modelos de negocio y de emprendedurismo.

\section{RESULTADOS}

Los resultados que a continuación se enuncian, muestran algunos elementos de fomento a la actitud emprendedora en la Institución, como parte de su propuesta educativa, y por el sentido de responsabilidad social que asume en la formación del estudiantado; que se da a través de dos vertientes:

1. Profesores. Se capacita a los profesores de las Comisiones Divisionales de la UJAT que apoyan el programa de emprendedores, por medio de talleres, diplomados y cursos de capacitación.

2. Alumnos. Además de recibir asesoramiento por parte de los profesores capacitados, se les ofrecen apoyos para eventos tales como: ferias de negocios y concursos como parte de las estrategias de motivación.

Como se aprecia, los posgrados entran en esta dinámica, pero el apoyo es incipiente, por los horarios en que se imparten las maestrías, y lo anterior está enfocado más a licenciaturas, careciéndose de una propuesta específica para los posgrados. A continuación, las siguientes gráficas, muestran los resultados de algunas preguntas realizadas en el cuestionario y que permiten tener una imagen del ambiente emprendedor de la unidad de Posgrado de la DACEA-UJAT.

Las siguientes gráficas son parte de los resultados obtenidos del análisis estadístico de datos. La gráfica 1 muestra que el 35\% de los entrevistados opinaron que al concluir sus estudios de posgrado, les gustaría emprender un negocio, como un deseo personal o para atender una necesidad social que haya identificado en su localidad, cualquiera que sea el motivo, la inquietud existe. El $25 \%$ señalaron la posibilidad de aplicar los conocimientos adquiridos en la maestría en su trabajo; el 22\% planean pedir un ascenso y el $18 \%$ quieren conseguir un empleo al finalizar sus estudios. 
FOMENTO DE LA ACTITUD EMPRENDEDORA, EN EL AMBIENTE UNIVERSITARIO PARA LA CREACIÓN DE MIPYMES. EL CASO DE POSGRADO DE LA DACEA-UJAT.

\section{Gráfica 1. Actividad a realizar al concluir el posgrado.}

¿Qué piensa hacer al concluir su programa de Maestría?

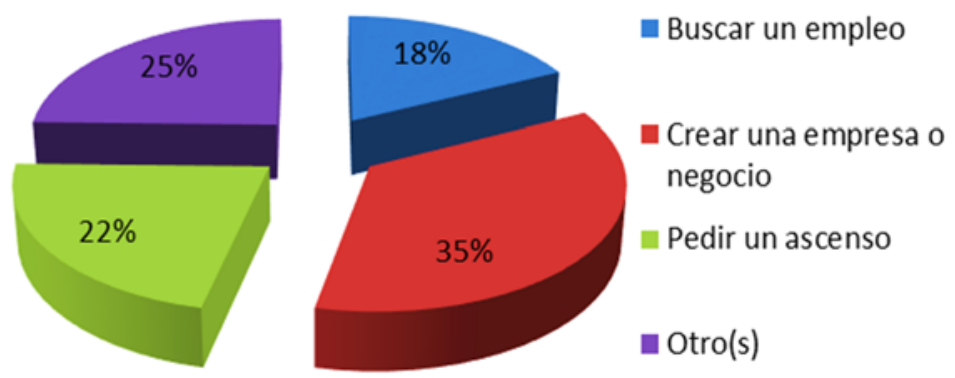

Fuente: Elaboración propia en base a los resultados de encuestas aplicadas.

El 95\% de los alumnos encuestados consideraron, que actualmente el Programa de Maestría de la UJAT, necesita reforzar las herramientas que ayuden a fortalecer la cultura del emprendimiento a nivel Posgrado. Por otro lado, el 5\% opinó que no es relevante, (Gráfica 2).

\section{Gráfica 2. Formación de Emprendedores en el posgrado.}

En la maestría, ¿considera que es importante tener formación de Emprendedores?

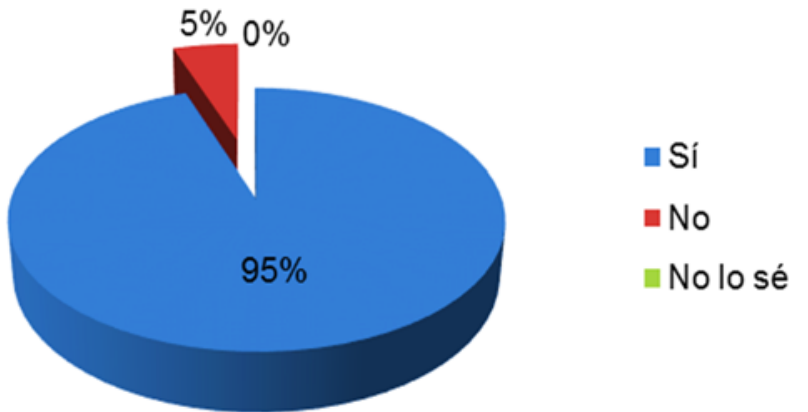

Fuente: Elaboración propia en base a los resultados de encuestas aplicadas. 
De los alumnos encuestados, el 78\% comentaron tener una idea de negocio. Mesa (2012), explica que las ideas surgen de y por diversas fuentes, sobre todo de estudiantes y universitarios, que la trasformación de las ideas debe estar acompañada por una serie de metodologías que hacen posible la evolución de estas hacia un nivel superior, pasando de idea a concepto de negocio. Es importante impulsar esas ideas para convertirlas en conceptos de negocio a través del lienzo de Osterwalder. El 22\% de los respondientes aseguraron que en este momento no tiene ideas de negocios, (ver gráfica 3).

Estos estudiantes manifiestan que para ellos es importante la creación de un negocio ya que significaría, un cambio positivo en su estilo de vida. Así lo señalaron un $88 \%$ de los encuestados.

Gráfica 3. Estudiantes de posgrado con ideas de negocio.

¿Tiene usted una idea de negocio?

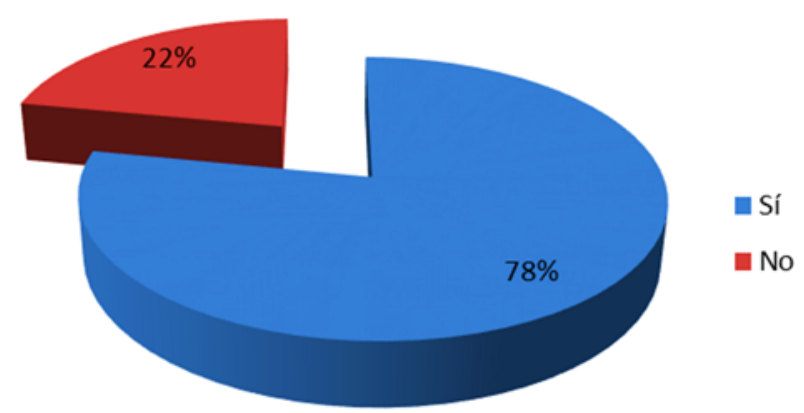

Fuente: Elaboración propia en base a los resultados de encuestas aplicadas. 
Hay diversos medios para impulsar la cultura del emprendimiento, el 61\%, los alumnos estarían dispuesto a pedir o recibir asesorías dentro del programa de Maestría para ver realizado su concepto de negocio. En la gráfica 4, al 92\% les gustaría tener apoyos que les ayuden a planificar una microempresa, lo que significa que se necesita trabajar con modelos de negocio para analizar el modo en que se crea, y los medios para llevarlo a cabo. $\mathrm{Y}$ el $8 \%$ aseguraron que no tiene ideas de negocio y por el momento no necesitan de apoyos.

\section{Gráfica 4. Necesidad de apoyos que ayuden a generar ideas} de negocios.

¿Le gustaría tener apoyos que le ayuden a generar ideas de negocio?

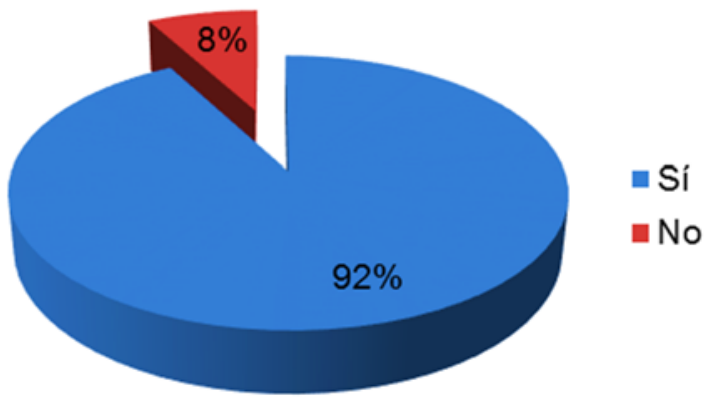

Fuente: Elaboración propia en base a los resultados de encuestas aplicadas.

\section{CONCLUSIONES}

El alumno de Posgrado de la DACEA-UJAT, requiere un proceso permanente de impulso y desarrollo, para propiciar la consolidación de una cultura emprendedora, donde se le fomente la creatividad e innovación en respuesta a problemas, necesidades y oportunidades en el Estado y que a través del lienzo de modelos de negocio de Osterwalder podrá encontrar también, el soporte para dar solución a estos fenómenos.

El papel que tiene la Dirección de Posgrado es seguir afianzando el conocimiento y el compromiso en los alumnos, promoviendo estrategias educativas que incentiven las actitudes emprendedoras entre los estudiantes. Seguir en la tarea de sensibilizarlos en temas de emprendimiento desde los inicios de su estancia en el programa; fortaleciendo a los que sueñan con transformar una idea en una MIPYME o los que piensan en reforzar 
su negocio. El Programa de Maestría debe continuar asumiendo el rol protagónico, alentando a quienes eligen la noble tarea de ser forjadores de su destino, -capaces de arriesgar, agregar valor, comprometidos y superando barreras-.

Los grandes desarrollos que se han dado en el mundo, han tenido como origen las universidades, es necesario que los alumnos de posgrado cuenten con estrategias para fomentar sus habilidades y trascender el espíritu emprendedor para la generación de empleos, y lograr con ello, la sinergia que necesita el programa de emprendedores y la unidad de posgrado, puesto que el objetivo es formar maestros con visión emprendedora. Y como consecuencia se logre el progreso económico, y el desarrollo social a través de una consolidación genuina de los emprendimientos creados. Los alumnos, deben aprovechar las oportunidades, y entender que el financiamiento no tiene por qué ser obstáculo para la ejecución de proyectos, más bien hay que comprender que es preciso instruirse y vincularse para ser mejores en lo que hacemos. 


\section{REFERENCIAS}

Anthony, J. \& Nitin, N. (2007). En su tiempo: lecciones de los grandes líderes del siglo XX. Recuperado de http://books.google.com

Cabrera, R. (2005). ¿Por qué es importante la capacitación? deGerencia.com Recuperado de http://www.degerencia.com/articulo/importante_capacitacion/

Diario Oficial de la Federación. (2013). Ley para el Desarrollo de la Competitividad de la Micro, Pequeña y Mediana Empresa. Recuperado de http://www.ordenjuridico.gob.mx/Documentos/Federal/wo83198.doc

Gutiérrez, G. (2011). Educación emprendedora en la universidad. Educando para el futuro. Recuperado de http://retos.ups.edu.ec/documents/1999140/2030551/Art3.pdf

Mesa, J. (2011). Concurso de Iniciativas Empresariales EAFIT 2012. Revista Iniciativas empresariales EAFIT. Recuperado de www.eafit.edu.co/concursoiniciativas

Mesa, J. (2012). Generación y captura de ideas: un reto para la innovación. Revista Catedras de innovacion empresarial. Recuperado de www.eafit.edu.co/catedrasdeinnovacion

Osterwalder, A. \& Pigneur, Y. (2011). Generación de modelos de negocios [Versión Adobe Digital Edition]. Recuperado de http://books.google.com

Ramírez, K. (2011). Análisis de libro teoría del desenvolvimiento económico, de Joseph A. Schumpeter. Contribuciones a la Economía. Recuperado de http://www.eumed.net/ce/2011a/

Stoner, J., Freeman, E. \& Gilbert, D. (1996). Administración (6a ed.). México: Pearson Educación. 